\title{
Effect of bone marrow mesenchymal stem cells on the polarization of macrophages
}

\author{
YUAN HUA ZHENG ${ }^{1}$, YI YU DENG ${ }^{2}$, WEN LAI $^{1}$, SHAO YI ZHENG ${ }^{1}$, HUI NING BIAN ${ }^{1}$, \\ ZU AN LIU ${ }^{1}$, ZHI FENG HUANG ${ }^{1}$, CHUAN WEI SUN ${ }^{1}$, HAN HUA LI ${ }^{1}$, HONG MIN LUO ${ }^{1}$, \\ LIANG HUA MA ${ }^{1}$, HAN XI CHEN $^{1}$ and BING XIONG ${ }^{1}$ \\ Departments of ${ }^{1}$ Burns and Wound Repair Surgery, and ${ }^{2}$ Critical Care and Emergency, Guangdong General Hospital, \\ Guangdong Academy of Medical Sciences, Guangzhou, Guangdong 510080, P.R. China
}

Received May 8, 2017; Accepted December 1, 2017

DOI: $10.3892 / \mathrm{mmr} .2018 .8457$

\begin{abstract}
Inflammation is a defensive response in the living tissue of the vascular system that acts against damage factors and involves various types of immune cells, including macrophages, neutrophils, endothelial cells and other associated immune molecules. If the release of inflammatory mediators is excessive, systemic inflammatory response syndrome may develop. Sepsis is the most common complication of severe burns and is a systemic inflammatory response syndrome that is caused by infectious factors and is capable of leading to multiple organ dysfunction and potentially death. Research concerning the mechanism and treatment of sepsis is crucial. Macrophages are an important type of immune cell that remove invasive pathogens and are involved in innate and adaptive immune responses. It has been previously reported that bone marrow mesenchymal stem cells (BMSCs) affect macrophages by regulating immunity. The present study aimed to investigate the effect of BMSCs on macrophage polarization in vivo and in vitro, in addition to the potential therapeutic effect of these cells on experimental sepsis. BMSCs and peritoneal macrophages were isolated from Sprague-Dawley rats and co-cultured overnight as a mixed culture or Transwell system, and subsequently stimulated with $100 \mathrm{ng} / \mathrm{ml}$ lipopolysaccharide (LPS). After $12 \mathrm{~h}$, the medium was replaced with normal complete medium for various durations and supernatants were collected to extract proteins and cells for ELISA, western blot and flow cytometry analysis to investigate different aspects of macrophages. Sepsis was induced in Sprague-Dawley rats by injection of LPS $(5 \mathrm{mg} / \mathrm{kg})$, followed by tail vein injection of BMSCs or PBS $1 \mathrm{~h}$ later. After 6, 12, 24 and 48 h, lung tissues
\end{abstract}

Correspondence to: Dr Bing Xiong, Department of Burns and Wound Repair Surgery, Guangdong General Hospital, Guangdong Academy of Medical Sciences, 106 Zhongshan Second Road, Yuexiu, Guangzhou, Guangdong 510080, P.R. China

E-mail: drxiongbing@163.com

Key words: sepsis, macrophages, bone marrow mesenchymal stem cells, polarization were harvested for pathological observation and peritoneal macrophages were collected for flow cytometry analysis to assess the expression of markers, including cluster of differentiation (CD)68 (used for gating), CD11c and CD206. The results demonstrated that, in the culture medium, LPS stimulation increased the expression of CD11c in macrophages, and the levels of tumor necrosis factor- $\alpha$ and inducible nitric oxide synthase were also increased. By contrast, in macrophages treated with BMSCs directly, the expression of CD11c was reduced compared with the LPS-stimulated macrophage alone group. However, the secretion of interleukin-10, transforming growth factor- $\beta$ and arginase- 1 was increased in the direct co-culture group, compared with the LPS-stimulated macrophage alone group. BMSCs reduced the inflammation in lung tissues and inhibited macrophage expression of CD11c in the rat model of sepsis. The results of the present study demonstrated that BMSCs co-cultured with macrophages directly inhibited macrophage differentiation into the M1 phenotype and reduced inflammation in macrophages stimulated by LPS. In vivo, BMSCs decreased the expression of CD11c in peritoneal macrophages and reduced the pathological inflammatory response in the lungs. The findings of the present study demonstrated that BMSCs may reduce the extent of the systemic inflammatory response, which may contribute to the development for a novel type of treatment for sepsis in the future.

\section{Introduction}

Sepsis is considered to be a systemic inflammatory response syndrome that occurs in response to severe infections, which includes pathogen invasion, extensive burns, severe trauma and shock (1). Recently, it has been demonstrated that sepsis is a multifaceted host response to infectious pathogens, which may be amplified by endogenous factors and is associated with a high morbidity and mortality (2). Although substantial developments have been made in the understanding of the basic pathogenesis of sepsis, no specific anti-sepsis treatments exist. As such, the management of patients relies mainly on early recognition allowing correct therapeutic measures to be conducted rapidly, including the administration of appropriate antibiotics, source control measures when necessary, 
resuscitation with intravenous fluids and vasoactive drugs when required (3). Research has indicated that admissions for sepsis are greater than those for myocardial infarction and stroke in the United States (4). In-hospital mortality remains high at $25-30 \%$ (3); therefore, it is necessary to develop a novel treatment for sepsis.

Within the hematopoietic system, macrophages are a one of the cell types that exhibit the highest plasticity, and they are present in every body tissue, where they trigger, regulate and affect the development of inflammation. Macrophages are differentiated into different phenotypes depending on the microenvironment (5-7). The classical activation phenotype (M1) and alternative activation phenotype (M2) express different surface makers, secrete different cytokines and have different roles in immunoregulation (7-9). Various studies concerning sepsis have demonstrated that macrophages are crucial participants in the systemic inflammatory response syndrome. Once activated, various soluble factors that directly and indirectly act on blood vessels, the immune system and other tissues are produced and secreted by macrophages, thus leading to a series of changes (10). However, excessive production of cytokines may lead to multiple organ failure and potentially death. Studies have reported that the development of sepsis is associated with the overactivation of M1 macrophages; excessive activation of M1 macrophages leads to the increased release of various inflammatory factors, including tumor necrosis factor (TNF)- $\alpha$, interleukin (IL)-1 and IL-6, subsequently resulting in severe inflammation (11-13).

Bone marrow mesenchymal stem cells (BMSCs) are a type of somatic stem cell $(14,15)$ that are isolated from bone myeloid tissue and exhibit self-renewal, unlimited proliferation and multipotent differentiation properties. Furthermore, BMSCs may be induced to differentiate into osteoblasts, adipocytes and chondrocytes in vitro. BMSCs have an important role in immunosuppression and also immunomodulation, in vitro and in vivo, in innate and adaptive immunity processes (16). A recent study demonstrated that human mesenchymal stem cells (MSCs) influence the process of inflammation by immunomodulating the expression of inflammatory cytokines from a variety of immune cells, including macrophages and altering the polarization of macrophages (17). The majority of previous studies have involved co-culture of RAW264.7 macrophage cells with different types of stem cells in vitro, followed by observation of the effects of stem cells on the polarization of macrophages $(18,19)$. However, there is no direct evidence as to whether stem cells have the ability to influence the resident peritoneal macrophages in vitro or alter the polarization of macrophages following injection into animals in vivo. Therefore, the present study investigated the effect of BMSCs on macrophage polarization in vitro and in vivo.

\section{Materials and methods}

Animals. All rats were maintained in specific-pathogen free environments at $20-26^{\circ} \mathrm{C}$ and $40-70 \%$ humidity under a $12 \mathrm{~h}$ light/dark cycle with, and free access to food and water. Rats were obtained from the Experimental Animal Center of the Sun Yat-sen University (license no. SCXK 2016-0029; Guangzhou, China). The animals were acclimatized for 3 days prior to initiating the experiment. A total of 70 male
Sprague-Dawley rats weighing $100 \pm 20$ g (4 weeks) were used for the isolation of BMSCs and macrophages; 36 male Sprague-Dawley rats weighing $130 \pm 20$ g (5 weeks) were used as the animal model. All experiments were performed following approval by the Ethics Committee of Guangdong General Hospital, Guangdong Academy of Medical Sciences (Guangzhou, China; approval no. GDREC2016254A).

Isolation and culture of rat BMSCs. BMSCs were obtained using adhesion to cell culture plastic as previously described (14). A total of 20 Sprague-Dawley rats, weighing $100 \pm 20 \mathrm{~g}$ (4 weeks), were euthanized via dislocation of the cervical vertebra and BMSCs were collected from the femur and tibia by flushing the shaft with chilled complete medium, which consisted of Dulbecco's modified Eagle's medium (DMEM, Gibco; Thermo Fisher Scientific, Inc., Waltham, MA, USA) supplemented with $10 \%$ fetal bovine serum (FBS; Gibco; Thermo Fisher Scientific, Inc.) and 1\% penicillin-streptomycin (Gibco; Thermo Fisher Scientific, Inc.). Cells were washed with medium three times and centrifuged at $4^{\circ} \mathrm{C}$ for $5 \mathrm{~min}$ at $192 \mathrm{x} \mathrm{g}$. The pellet was resuspended and cultured in $25 \mathrm{~cm}^{2}$ culture flasks with complete medium at $37^{\circ} \mathrm{C}$ and $5 \% \mathrm{CO}_{2}$ in air. BMSCs preferentially attached to polystyrene surfaces, and, after $72 \mathrm{~h}$, non-adherent cells were discarded. Fresh medium was added and replaced every 2-3 days. When cells reached $80-90 \%$ confluence, the adherent cells were trypsinized with $0.25 \%$ trypsin-EDTA (Invitrogen; Thermo Fisher Scientific, Inc.). Following three cell passages, the expression of cell surface markers, including cluster of differentiation (CD)29, CD90, CD45 and CD44 (Table I), were analyzed by flow cytometry, which is described in a later paragraph. Additionally, cells were induced to differentiate into osteoblasts and adipocytes, to determine the purity of the BMSC population. For standard osteogenic differentiation, confluent monolayers of BMSCs were incubated in medium supplemented with $0.01 \%$ dexamethasone, $0.2 \%$ ascorbic acid, $1 \%$ glutamine and $1 \% \beta$-glycerol phosphate (Cyagen Biosciences, Inc., Guangzhou, China). Culture medium was replaced every 3 days. After 30 days, the culture medium was fixed with $4 \%$ paraformaldehyde for $30 \mathrm{~min}$ at room temperature and incubated with Alizarin Red (Cyagen Biosciences, Inc.) for $30 \mathrm{~min}$ at room temperature. Excess stain was removed by washing four times with PBS. For standard adipogenic differentiation, confluent monolayers of BMSCs were incubated in medium supplemented with $1 \%$ glutamine, $0.1 \% 3$-isobutyl-1-methylxanthine, $0.1 \%$ rosiglitazone and $0.2 \%$ insulin (Cyagen Biosciences, Inc.) with medium changed every 1-3 days. After 30 days, the culture medium was fixed with $4 \%$ paraformaldehyde for $30 \mathrm{~min}$ at room temperature and incubated with Oil Red $\mathrm{O}$ for $30 \mathrm{~min}$ at room temperature. BMSCs were collected for experiments in the third passage via trypsinization and resuspension in PBS to a concentration of $2 \times 10^{6}$ cells $/ \mathrm{ml}$ immediately prior to injection; cells were observed under a light microscope.

Isolation and culture of rat peritoneal macrophages. A total of 50 male Sprague-Dawley rats, weighing $100 \pm 20 \mathrm{~g}$ (4-weeks-old) used for obtaining peritoneal macrophages. PBS $(20 \mathrm{ml})$ supplemented with $10 \%$ FBS was injected into the peritoneal cavity and the rat abdomen was gently massaged 
Table I. Details of antibodies used for flow cytometry and western blotting.

\begin{tabular}{lllll}
\hline Antibody & Source & Dilution & & Manufacturer \\
CD29-FITC & Hamster & $1: 20$ & BD Biosciences & Catalogue number \\
CD44-FITC & Mouse & $1: 20$ & BD Biosciences & 555005 \\
CD45-FITC & Mouse & $1: 20$ & BD Biosciences & 550974 \\
CD90-PE & Mouse & $1: 20$ & BD Biosciences & 554877 \\
CD11b-APC & Mouse & $1: 20$ & BD Biosciences & 554898 \\
CD68-Alexa Fluor 488 & Mouse & $1: 20$ & Bio-Rad Laboratories, Inc. & 562102 \\
CD11c-Alexa Fluor 647 & Mouse & $1: 20$ & Bio-Rad Laboratories, Inc. & MCA341A488 \\
CD206 & Rabbit & $1: 20$ & Abcam & MCA1441A647 \\
iNOS & Mouse & $1: 1,000$ & Abcam & ab64693 \\
Arg-1 & Rabbit & $1: 1,000$ & Abcam & ab49999 \\
F(ab')2 donkey anti-rabbit IgG-PE & Donkey & $1: 100$ & eBioscience; Thermo Fisher Scientific, Inc. & $12-4739$ \\
Anti-rabbit IgG-HRP & Goat & $1: 5,000$ & Santa Cruz Biotechnology, Inc. & sc-2004 \\
Anti-mouse IgG-HRP & Goat & $1: 5,000$ & Santa Cruz Biotechnology, Inc. & sc-2005 \\
$\beta$-actin & Mouse & $1: 5,000$ & Santa Cruz Biotechnology, Inc. & sc-47778
\end{tabular}

BD, Biosciences, Franklin Lakes, NJ, USA; Bio-Rad Laboratories, Inc., Hercules, CA, USA; Abcam, Cambridge, MA, USA; Thermo Fisher Scientific, Inc., Waltham, MA, USA; Santa Cruz Biotechnology, Inc., Dallas, TX, USA. FITC, fluorescein isothiocyanate; PE, phycoerythrin; APC, allophycocyanin; iNOS, inducible nitric oxide synthase; Arg-1, arginase-1; HRP, horseradish peroxidase.

for $5 \mathrm{~min}$ (20). Macrophages were isolated from the peritoneal cavity by collecting as much peritoneal fluid as possible, and the collected fluid was centrifuged at $4^{\circ} \mathrm{C}$ for $5 \mathrm{~min}$ at $192 \mathrm{x} \mathrm{g}$ to pellet the cells. The pellet was resuspended and cultured in 6-well plates with complete medium at $37^{\circ} \mathrm{C}$ and $5 \% \mathrm{CO}_{2}$ in air. After $6 \mathrm{~h}$, non-adherent cells were discarded. Fresh medium was added and replaced every 1-2 days. After 2 days, adherent cells were harvested and purified using CD68 and CD11b (Table I) via flow cytometry, which is described in a later paragraph. These peritoneal macrophages were collected for culturing with BMSCs in vitro.

Co-culture of peritoneal macrophages with BMSCs in vitro. Initially, peritoneal macrophages were incubated in 6-well plates (Corning Inc., Corning, NY, USA) at a concentration 100,000 cells per $2 \mathrm{ml}$ at $37^{\circ} \mathrm{C}$. After $12 \mathrm{~h}$, the culture (complete) medium was removed and either 100,000 BMSCs in fresh medium or medium alone was added to each well. Macrophages were divided into four groups according to different treatments: Macrophages cultured in normal medium for $96 \mathrm{~h}$; macrophages incubated with lipopolysaccharide (LPS; 100 ng/ml; Sigma-Aldrich, Merck KGaA, Darmstadt, Germany) for $12 \mathrm{~h}$ followed by culture in normal medium for $84 \mathrm{~h}$; macrophages co-cultured with BMSCs, separated by a Transwell insert $(0.4 \mu \mathrm{m}$ pore size polyester membrane; Corning, Inc.), were incubated with LPS $(100 \mathrm{ng} / \mathrm{ml})$ for $12 \mathrm{~h}$ followed by culture in normal medium for $84 \mathrm{~h}$; and a direct co-culture of macrophages and BMSCs attached to the same substrate was incubated with LPS (100 ng/ml) for $12 \mathrm{~h}$ followed by culture in normal medium for $84 \mathrm{~h}$. All cell incubations were conducted at $37^{\circ} \mathrm{C}$ and $5 \% \mathrm{CO}_{2}$. Subsequently, cell culture supernatants, protein and cells were collected for ELISA, western blotting and flow cytometry analysis, respectively, to investigate the effects of co-culture on macrophages.
LPS model of sepsis. Male Sprague-Dawley rats, weight $130 \pm 20 \mathrm{~g}$ (5-weeks-old) were fed a standard diet and acclimatized in a quiet room for three days prior to experimentation. Rats were administered $5 \mathrm{mg} / \mathrm{kg}$ LPS dissolved in PBS via tail vein injection (21). All animals were randomly divided into three groups according to different treatments: Normal control group $(n=4)$, LPS-stimulated group $(n=16)$ and BMSC-treated group $(n=16)$ by the random number table. The normal control group was injected with $1 \mathrm{ml}$ PBS $(\mathrm{n}=4)$, LPS-stimulated group was injected with $1 \mathrm{ml} \mathrm{LPS}(5 \mathrm{mg} / \mathrm{kg})$ dissolved in PBS ( $\mathrm{n}=16)$. The BMSC-treated group was injected with LPS $(5 \mathrm{mg} / \mathrm{kg})$ and after $1 \mathrm{~h}$, injected with BMSCs ( $2 \times 10^{6}$ cells in $1 \mathrm{ml}$ PBS). Animals in the normal control group were euthanized to harvest their lungs for hematoxylin-eosin staining after $24 \mathrm{~h}$, while lungs from animals in other groups were obtained after $6,12,24,48 \mathrm{~h}$. In addition, rat peritoneal macrophages were obtained by peritoneal injection of DMEM after LPS stimulation for $24 \mathrm{~h}$ in the normal control group, while the procedure was performed after LPS stimulation for 24 and $48 \mathrm{~h}$ in the LPS-stimulated group and BMSC-treated group. Macrophages were isolated from the peritoneal cavity by collecting as much peritoneal fluid as possible, and the collected fluid was centrifuged at $4^{\circ} \mathrm{C}$ for $5 \mathrm{~min}$ at $192 \mathrm{x}$ g to pellet the cells. The pellet was resuspended and cultured in 6-well plates, with complete medium, at $37^{\circ} \mathrm{C}$ and $5 \% \mathrm{CO}_{2}$ in air. After $6 \mathrm{~h}$, non-adherent cells were discarded. Medium was replaced with fresh medium. After $24 \mathrm{~h}$, the adherent cells were harvested to analyze the expression of CD68, CD11c and CD206 markers (Table I) by flow cytometry at the Sun Yat-sen Memorial Hospital of Sun Yat-sen University, as described in a later paragraph.

Histopathological examination of lungs. Whole lung samples were fixed in $10 \%$ formalin for $24 \mathrm{~h}$ at room temperature and 
were sent to Google Biological Technology Co., Ltd. (Wuhan, China) for further procedure, where they were subjected to conventional methods of dehydration, paraffin-embedding and hematoxylin and eosin (H\&E) staining. All the lung tissues (thickness: $5 \mu \mathrm{m}$ ) were observed with an inverted fluorescence microscope (TI-S; Nikon Corporation, Tokyo, Japan).

Flow cytometry analysis of cell markers in vitro and in vivo. Flow cytometry was used to assess the cell surface or intracellular marker, CD68, of BMSCs and macrophages, as described in previous literature (22-26). Adherent cells $\left(2 \times 10^{5}\right.$ cells/centrifuge tube) were lightly trypsinized, then blocked with complete medium for $2 \mathrm{~min}$ at room temperature, harvested and incubated with the specific primary and secondary antibodies for $30 \mathrm{~min}$ at $4^{\circ} \mathrm{C}$ in the dark. The following antibodies were used for BMSCs: Anti-CD29, anti-CD44, anti-CD45 and anti-CD90 (Table I). Similarly, the following antibodies for macrophages were used: Anti-CD11b and anti-CD68 (Table I). The following antibodies were used for macrophages that were collected from the in vitro co-culture system or in vivo animal model: Anti-CD206, F(ab')2 donkey anti-rabbit IgG-phycoerythrin, anti-CD11c and anti-CD68 (Table I). Specifically, cells were fixed and permeabilized using a FIX \& PERM ${ }^{\mathrm{TM}}$ Cell Permeabilization kit (Invitrogen; Thermo Fisher Scientific, Inc.) prior to the addition of anti-CD68. The recommended isotype controls for each fluorochrome were used. Following incubation, cells were washed with PBS and analyzed using a FACSVerse flow cytometer and FACSuite (BD Biosciences, Franklin Lakes, NJ, USA).

Western blot analysis. For protein extraction in vitro, following direct or Transwell-separated co-culture of macrophages with BMSCs for $48 \mathrm{~h}$, including an initial $12 \mathrm{~h}$ period of LPS treatment, cells were washed with ice-cold PBS and lysed in radioimmunoprecipitation assay buffer (BestBio Science, Shanghai, China) containing a protease inhibitor cocktail. After $30 \mathrm{~min}$, cell lysates were collected and centrifuged at 13,400 x $\mathrm{g}$ for $20 \mathrm{~min}$ at $4^{\circ} \mathrm{C}$; the supernatant was prepared as a protein extract. The supernatant was measured for protein concentration by a Bicinchoninic Acid Protein assay kit (Bioworld Technology, Inc., St. Louis Park, MN, USA). A total of $20 \mu \mathrm{g}$ protein was separated by $10 \%$ SDS-PAGE Bis-Tris gels and transferred to polyvinylidene fluoride membranes (Bio-Rad Laboratories, Inc., Hercules, CA, USA) in ice-cold water for $2 \mathrm{~h}$. The membranes were washed three times with Tris-buffered saline with Tween-20 (TBST) for $5 \mathrm{~min}$ and then blocked with 5\% skimmed milk powder dissolved in TBST for $2 \mathrm{~h}$ at room temperature. Membranes were subsequently incubated with primary antibodies against inducible nitric oxide synthase (iNOS), arginase-1 (Arg-1) and $\beta$-actin for $16 \mathrm{~h}$ at $4^{\circ} \mathrm{C}$ (Table I). The following horseradish peroxidase (HRP)-conjugated secondary antibodies were used: Goat anti-rabbit $\operatorname{IgG}$ and goat anti-mouse $\mathrm{IgG}$ for $3 \mathrm{~h}$ at room temperature (Table I). Protein expression was detected using Biodlight ${ }^{\mathrm{TM}}$ ECL chemiluminescent HRP Substrate (Bioworld Technology, Inc.) and an ImageQuant LAS 500 imaging system (GE Healthcare Life Sciences, Buckinghamshire, UK). Blots against $\beta$-actin served as the loading control. The amount of target protein calculated by gray scanning via Image J v1.39 software (National Institutes of Health Bethesda, MD, USA).
Analysis of cytokine production by ELISA. In vitro, cell-free supernatants were collected and centrifuged at 13,400 x $\mathrm{g}$ for $30 \mathrm{~min}$ at $4^{\circ} \mathrm{C}$ of each group at $3,7,12,24,48,72 \mathrm{~h}$ after LPS stimulation and assayed for the concentration of TNF- $\alpha$, transforming growth factor (TGF)- $\beta$ and IL-10 with ELISA kits (rat IL-10, DKW12-3100-096; rat TNF- $\alpha$ DKW12-3720-096 and rat TGF- $\beta$, DKW12-3710-096; Dakewe Bioengineering Co., Ltd., Shenzhen, China). The optical density was measured at $450 \mathrm{~nm}$ using a microplate reader (Multiskan GO; Thermo Fisher Scientific, Inc.) with the correction wavelength set at $620 \mathrm{~nm}$.

Statistical analysis. Data are presented as the mean \pm standard error of the mean from at least three independent experiments. According to the characteristics of the data, different statistical methods were used for analysis. The differences between groups were analyzed with one-way analysis of variance followed by Fisher's least significant difference or Dunnett's post-hoc tests, and Nemenyi test, which was performed to analyze the results for the percentages of CD206 and western blot analysis as a post hoc test following analysis via the Kruskall-Wallis test. SPSS 16.0 software (SPSS, Inc., Chicago, IL, USA) was used to perform statistical analysis. GraphPad Prism 5.0 software was used to produce graphs (GraphPad Software, Inc., La Jolla, CA, USA). P $<0.05$ was considered to indicate a statistically significant difference.

\section{Results}

Characteristics of macrophages and BMSCs. The cell surface antigen phenotype of macrophages and BMSCs isolated from rats was assessed by flow cytometry. Macrophages expressed both CD68 (100\%) and CD11b (94.07\%), while BMSCs expressed high levels of CD44 (95.15\%), CD29 (99.98\%) and CD90 (99.41\%), and were considered negative for expression of CD45 (0.63\%; data not shown). In addition, staining with Alizarin Red and Oil Red O demonstrated that BMSCs obtained from rats successfully differentiated into osteogenic and adipogenic mesenchymal lineages, respectively (Fig. 1).

Co-culture of macrophages with BMSCs blocks LPS-induced macrophage polarization to the MI phenotype. Flow cytometry analysis with a CD68 gating strategy demonstrated that there was a high expression level of CD11c in $90.00 \pm 4.03 \%$ of LPS-stimulated peritoneal macrophages, compared with unstimulated macrophages (Fig. 2A and B; Table II), indicating that the majority of macrophages in the LPS-stimulated group were M1 macrophages. Exposure of macrophages to LPS, while being indirectly co-cultured with BMSCs in a Transwell, resulted in macrophages acquiring the M1 phenotype, with $86.79 \pm 0.69 \%$ of the macrophages expressing a high level of CD11c (Fig. 2C; Table II). However, when peritoneal macrophages co-cultured directly with BMSCs on the same substrate were exposed to LPS, a lower level of CD11c expression was observed among macrophages, with $66.21 \pm 3.19 \%$ (Fig. 2D; Table II). The expression of CD206 in the different groups was low and there was no significant difference among the groups (Fig. 2; Table II).

Macrophage co-culture with BMSCs reduces LPS-induced cytokine release. Levels of TNF- $\alpha$, IL-10 and TGF- $\beta$ were 

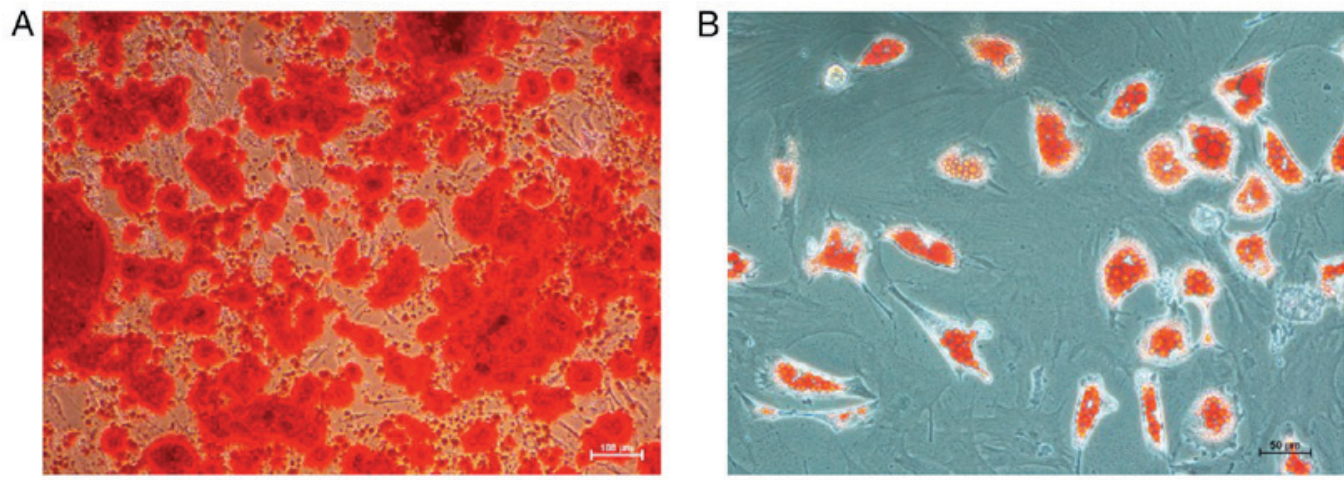

Figure 1. Differentiation of BMSCs into osteogenic and adipogenic cells. (A) Culture of BMSCs in osteogenic differentiation medium indicating the formation of calcium-containing precipitates stained by Alizarin Red. Scale bar, $100 \mu \mathrm{m}$; magnification, x100. (B) Culture of BMSCs in adipocyte differentiation medium indicates cells containing fat droplets, as revealed by Oil Red O staining. Scale bar, $50 \mu \mathrm{m}$; magnification, x200. BMSCs, bone marrow mesenchymal stem cells.
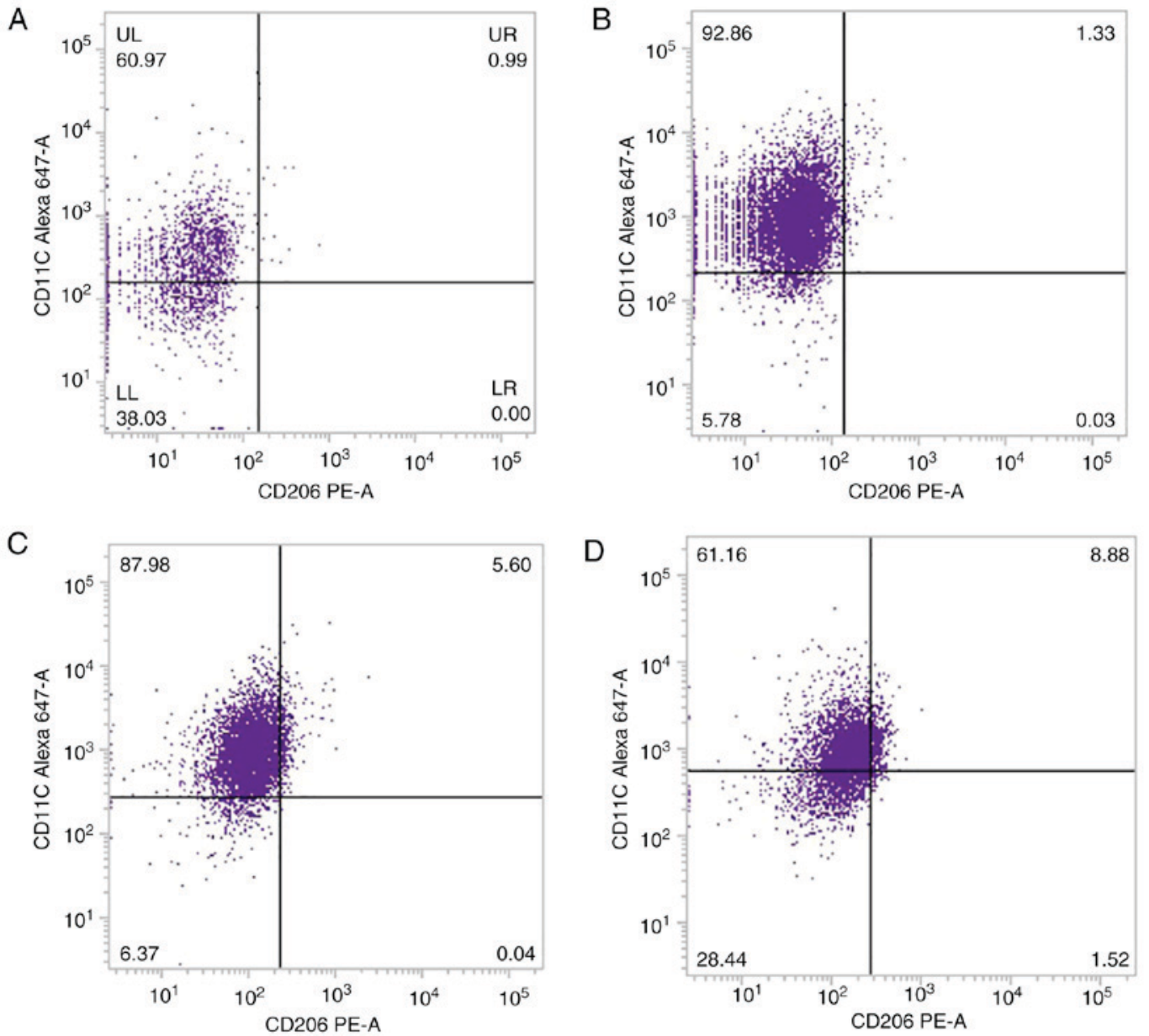

Figure 2. Representative flow cytometry scatter plots of peritoneal macrophage CD11c and CD206 expression following exposure to LPS in vitro. (A) Expression of CD11c and CD206 in the normal control group. (B) Expression of CD11c and CD206 in macrophages stimulated with LPS. (C) Expression of CD11c and CD206 in macrophages co-cultured with BMSCs in a Transwell and stimulated with LPS. (D) Expression of CD11c and CD206 in macrophages directly co-cultured with BMSCs and stimulated with LPS. LPS, lipopolysaccharide; BMSCs, bone marrow mesenchymal stem cells; Alexa 647, Alexa Fluor 647; PE, phycoerythrin.

quantified by ELISA in cell culture supernatants. LPS stimulated macrophages to produce high levels of TNF- $\alpha$, compared with the control group (Fig. 3A). No differences in TNF- $\alpha$ release were observed among LPS-stimulated macrophage alone, Transwell co-culture with BMSCs and direct co-culture with BMSCs groups at 3,7 and $12 \mathrm{~h}$ following LPS addition.
However, after 24, 48 and 72 h, TNF- $\alpha$ levels were decreased when macrophages were directly co-cultured with BMSCs, compared with the LPS-stimulated macrophage alone group (Fig. 3A). IL-10 levels were increased markedly at 7, 12, 48 and $72 \mathrm{~h}$ following LPS stimulation, compared with the control group, with levels particularly high when macrophages were 
Table II. Percentage of CD11c-positive and CD206-postive macrophages in the different groups in vitro.

\begin{tabular}{lll}
\hline Group & CD11c, $\%$ & CD206, \% \\
\hline M & $53.48 \pm 5.43$ & $0.61 \pm 0.27$ \\
ML & $90.00 \pm 4.03^{\mathrm{a}, \mathrm{b}}$ & $0.03 \pm 0.02$ \\
MT & $86.79 \pm 0.69^{\mathrm{a}, \mathrm{b}}$ & $0.03 \pm 0.15$ \\
MM & $66.21 \pm 3.19$ & $0.57 \pm 0.31$ \\
\hline
\end{tabular}

For CD11c, $\mathrm{F}=21.32$ and $\mathrm{P}<0.01$. For $\mathrm{CD} 206, \chi^{2}=6.99$ and $\mathrm{P}=0.07$. ${ }^{a} \mathrm{P}<0.05$ vs. $\mathrm{M}$ group and ${ }^{\mathrm{b}} \mathrm{P}<0.05$ vs. $\mathrm{MM}$ group, $\mathrm{n}=5$ per group. LPS, lipopolysaccharide; BMSCs, bone marrow mesenchymal stem cells; M, macrophages cultured in normal medium for $96 \mathrm{~h}$; ML, macrophages undergoing a $12 \mathrm{~h}$ incubation with LPS $(100 \mathrm{ng} / \mathrm{ml})$ followed by culture in normal medium for $84 \mathrm{~h}$; MT, macrophages co-cultured with BMSCs, separated in a Transwell, undergoing a $12 \mathrm{~h}$ incubation with LPS followed by culture in normal medium for $84 \mathrm{~h}$; $\mathrm{MM}$, macrophages and BMSCs directly co-cultured, attached to the same substrate, undergoing a $12 \mathrm{~h}$ incubation with LPS followed by culture in normal medium for $84 \mathrm{~h}$.

co-cultured with BMSCs (Fig. 3B). TGF- $\beta$ was induced in the group that consisted of direct co-culture of macrophages and BMSCs stimulated by LPS, but not in the Transwell co-culture of macrophages with BMSCs (Fig. 3C). These results indicate that co-culture of macrophages with BMSCs may lead to the inhibition of proinflammatory cytokine production and stimulation of anti-inflammatory factor production (Fig. 3).

BMSCs reciprocally regulate iNOS and Arg-1 in activated macrophages. iNOS and Arg-1 are markers of the M1 and M2 phenotypes, respectively. The present study analyzed the protein expression of iNOS and Arg-1 by western blot analysis in the four groups in vitro. The results demonstrated that iNOS expression was increased in macrophages following LPS stimulation, which was markedly decreased by direct co-culture with BMSCs (Fig. 4). By contrast, Arg-1 was markedly induced in macrophages following direct co-culture with BMSCs, compared with the other three groups. These results demonstrated that LPS stimulation of macrophages alone induced the expression of iNOS, while the presence of BMSCs caused macrophages to polarize towards an M2 phenotype following LPS stimulation (Fig. 4).

BMSC administration reduces LPS-induced sepsis in vivo. The effect of BMSCs in the septic rat model induced by LPS $(5 \mathrm{mg} / \mathrm{kg})$ via tail vein injection was also investigated. At $1 \mathrm{~h}$ after injection of LPS, rats in the BMSC-treated group were injected with $1 \mathrm{ml}$ BMSCs $\left(2 \times 10^{6}\right.$ cells $\left./ \mathrm{ml}\right)$. Subsequently, lung tissues from LPS-stimulated and BMSC-treated groups were collected at 6,12, 24 and $48 \mathrm{~h}$ for histopathological examination, while lung tissues from the normal control group were only collected at $24 \mathrm{~h}$ after injection with LPS. Lung tissues in the normal control group at $24 \mathrm{~h}$ after injection of LPS exhibited normal and complete alveoli without congestion or inflammatory cell infiltration. Histological assessment of representative lung sections from rats with LPS-induced sepsis highlighted a large degree of inflammatory cell infiltration,
A
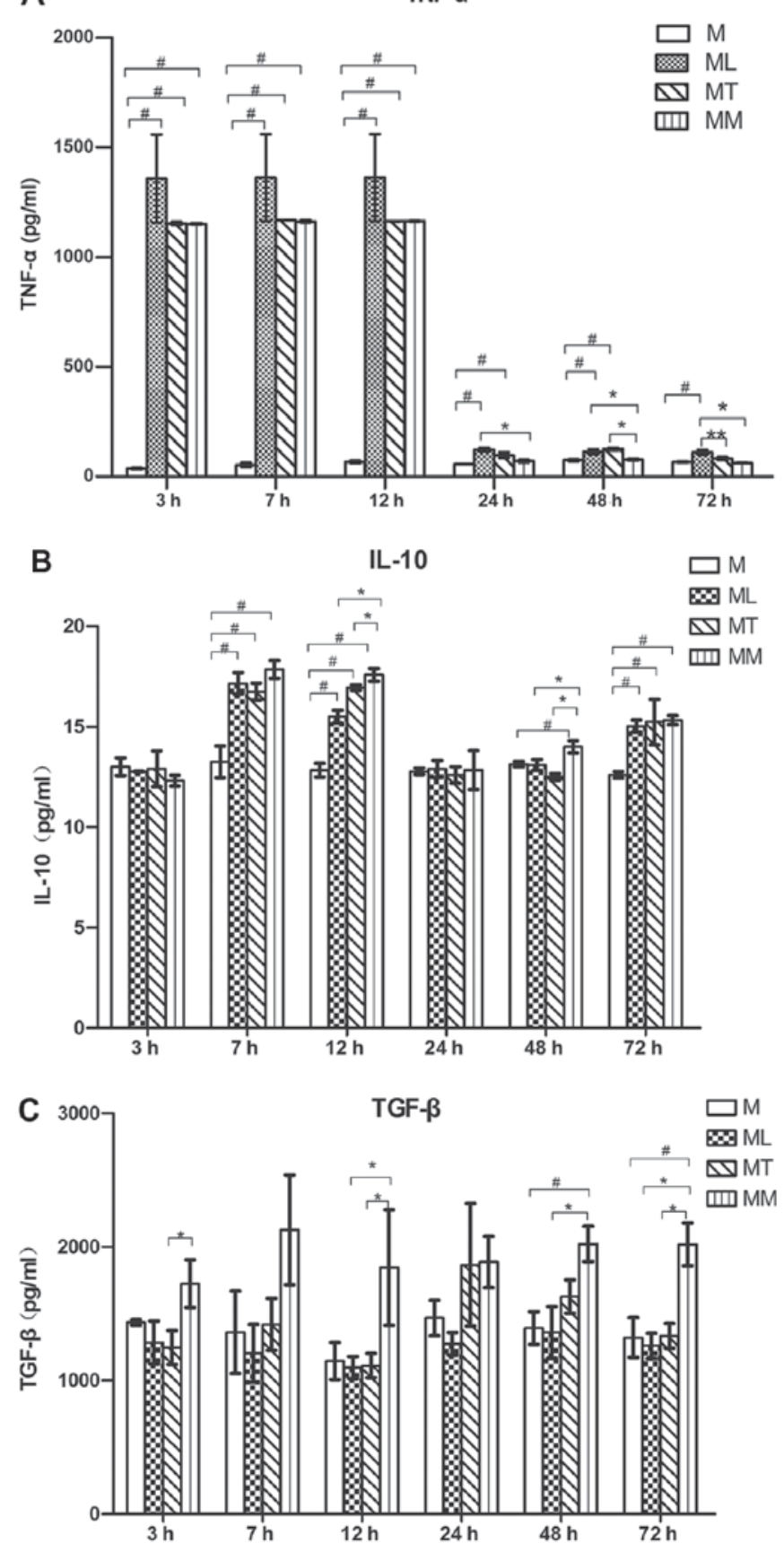

Figure 3. Secretion levels of TNF- $\alpha$, IL-10 and TGF- $\beta$ in cell culture medium were measured by ELISA. (A) Direct co-culture of with BMSCs inhibited the production of the proinflammatory cytokine TNF- $\alpha$ in rat peritoneal macrophages. Direct co-culture with BMSCs enhanced the production of (B) IL-10 and (C) TGF- $\beta$ in peritoneal macrophages. Results are presented as the mean \pm standard error of the mean, $\mathrm{n}=3$ per group. ${ }^{~} \mathrm{P}<0.05$ vs. $\mathrm{M}$ group, ${ }^{* *} \mathrm{P}<0.05$ vs. MT group and ${ }^{*} \mathrm{P}<0.05$ vs. MM group, as indicated. TNF, tumor necrosis factor; IL, interleukin; TGF, transforming growth factor; BMSCs, bone marrow mesenchymal stem cells; $\mathrm{M}$, macrophages cultured in normal medium for $72 \mathrm{~h}$; ML, macrophages undergoing a $12 \mathrm{~h}$ incubation with LPS $(100 \mathrm{ng} / \mathrm{ml})$ followed by culture in normal medium for $60 \mathrm{~h}$; MT, macrophages co-cultured with BMSCs, separated in a Transwell, undergoing a $12 \mathrm{~h}$ incubation with LPS followed by culture in normal medium for $60 \mathrm{~h}$; MM, macrophages and BMSCs directly co-cultured, attached to the same substrate, undergoing a $12 \mathrm{~h}$ incubation with LPS followed by culture in normal medium for $60 \mathrm{~h}$.

serious congestion and pulmonary interstitial thickening at $12 \mathrm{~h}$ after LPS administration. However, the inflammatory response detected in lung tissues was decreased by injection 
A
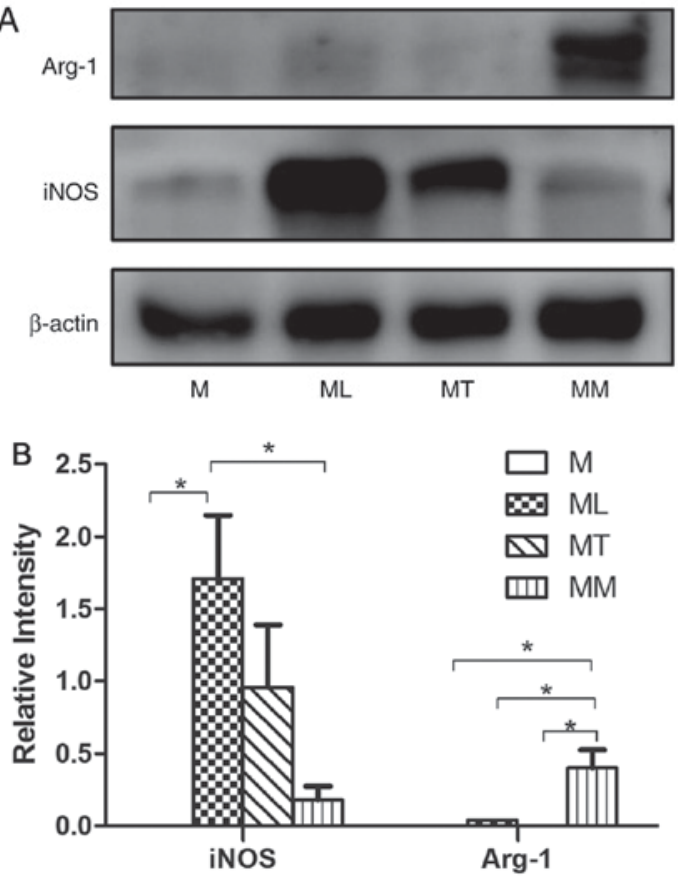

Figure 4. Western blot analysis of iNOS and Arg-1 protein expression following co-culture of peritoneal macrophages with BMSCs. (A) Representative western blot bands for iNOS, Arg-1 and $\beta$-actin in each group. (B) Densitometric analysis was performed to quantify protein expression. BMSCs inhibited LPS-induced induction of iNOS and enhanced Arg-1 expression in peritoneal macrophages. $\mathrm{n}=5$ per group; ${ }^{*} \mathrm{P}<0.05$ as indicated. iNOS, inducible nitric oxide synthase; Arg-1, arginase-1; BMSCs, bone marrow mesenchymal stem cells; LPS, lipopolysaccharide; M, macrophages cultured in normal medium for $48 \mathrm{~h}$; ML, macrophages undergoing a $12 \mathrm{~h}$ incubation with LPS $(100 \mathrm{ng} / \mathrm{ml})$ followed by culture in normal medium for $36 \mathrm{~h}$; MT, macrophages co-cultured with BMSCs, separated in a Transwell, undergoing a $12 \mathrm{~h}$ incubation with LPS followed by culture in normal medium for $36 \mathrm{~h}$; MM, macrophages and BMSCs directly co-cultured, attached to the same substrate, undergoing a $12 \mathrm{~h}$ incubation with LPS followed by culture in normal medium for $36 \mathrm{~h}$.

of BMSCs, as tissues exhibited relatively normal alveoli accompanied by a small degree of inflammatory cell infiltration, and marginal congestion and pulmonary interstitial thickening. The pathological observations at 12 and $24 \mathrm{~h}$ in the LPS-stimulated and BMSCs-treated groups were similar. At $48 \mathrm{~h}$, the inflammatory response was marginally improved in the LPS-simulated group, with inflammatory cell infiltration, congestion, while pulmonary interstitial thickening was decreased compared with at 12 and $24 \mathrm{~h}$ in the LPS-stimulated group. Alterations in the BMSC-treated group returned to normal levels without congestion or inflammatory cell infiltration (Fig. 5).

Flow cytometry analysis of cell markers in LPS-induced sepsis in rats. In order to confirm our hypothesis that BMSCs may enhance the anti-inflammatory phenotype and inhibit the proinflammatory phenotype of macrophages, the present study investigated CD11c and CD206 expression on the surface of macrophages isolated from septic rats. The ratio of macrophages that expressed CD11c in the LPS-stimulated group was similar to the ratio in the BMSC-treated group at $24 \mathrm{~h}$ after LPS injection ( $\mathrm{P}>0.05$; Table III; Fig. 6), while it was significantly higher compared with the normal control group $(\mathrm{P}<0.01$; Table III; Fig. 6$)$. Compared with the normal control group, the ratio of $\mathrm{CD} 11 \mathrm{c}$-positive macrophages was
Table III. Percentage of CD11c-positive and CD206-positive macrophages in the different groups in vivo.

\begin{tabular}{lcc}
\hline Group & CD11c, \% & CD206, \% \\
\hline Normal control group & $50.90 \pm 4.94$ & $0.22 \pm 0.12$ \\
LPS, 24 h & $87.43 \pm 2.24^{\mathrm{a}}$ & $0.11 \pm 0.10$ \\
LPS+BMSCs, 24 h & $86.82 \pm 3.97^{\mathrm{a}}$ & $0.03 \pm 0.02$ \\
LPS, 48 h & $61.40 \pm 5.57^{\mathrm{b}}$ & $1.83 \pm 0.28$ \\
LPS+BMSCs, 48 h & $23.07 \pm 4.64^{\mathrm{a}}$ & $0.61 \pm 0.56$ \\
\hline
\end{tabular}

For CD11c, $\mathrm{F}=37.10$ and $\mathrm{P}<0.01$. For $\mathrm{CD} 206, \chi^{2}=9.46$ and $\mathrm{P}=0.05$. ${ }^{\mathrm{a}} \mathrm{P}<0.01 \mathrm{vs}$. normal control group and ${ }^{\mathrm{b}} \mathrm{P}<0.01$ vs. LPS+BMSCs group at $48 \mathrm{~h}, \mathrm{n}=4$ per group. LPS, lipopolysaccharide; BMSCs, bone marrow mesenchymal stem cells.

marginally increased in the LPS-stimulated group at $48 \mathrm{~h}$ after LPS injection ( $\mathrm{P}>0.05$; Table III; Fig. 6 ), while it was significantly higher compared with the BMSC-treated group at $48 \mathrm{~h}$ after LPS injury (P<0.01; Table III; Fig. 6). The ratio of CD206-positive macrophages was similar in each group, with no significant differences among the groups (Table III; Fig. 6).

\section{Discussion}

The pathophysiology of sepsis involves complex interactions between microbial pathogens and the host immune system to regulate inflammation and coagulation (27). The immune and neurohumoral systems closely control inflammation under normal circumstances and eliminate pathogen invasion. When microorganisms or microbial products invade the body and macrophages are polarized into the M1 phenotype, excessive levels of proinflammatory factors, including TNF- $\alpha$, IL- $1 \beta$ and IL-6, are produced, which results in a cascade of inflammatory responses and leads to systemic inflammation, severe sepsis and potentially septic shock (28-31). Therefore, it is important that macrophage phenotype is regulated and the excessive activation of M1 macrophages is avoided in sepsis. Due to their immunoregulatory effects, BMSCs have wide application potential in tissue engineering. BMSCs have been reported to regulate immune cells, including mononuclear macrophages, and control excessive inflammatory reactions (32).

The current study investigated the effects of BMSCs on the polarization of macrophages in vitro and in vivo. Identification of BMSCs by flow cytometry demonstrated that the percentage of cells expressing BMSC surface marker characteristics was consistent with the definition of BMSCs provided by the International Society of Cellular Therapy $(22,23)$. Furthermore, the results of the present study also confirmed the BMSCs were capable of differentiating into osteoblast and adipocyte phenotypes, indicating that BMSCs have the capacity to differentiate into mesenchymal lineages under the appropriate conditions in vitro. In the macrophage system of the present study, $>90 \%$ of cells expressed the macrophage markers CD11b and CD68. These results indicated that the purity of BMSCs and macrophages in the current study complied with the essential requirements for our experiments. According to previous literature, macrophages express a variety of markers. 

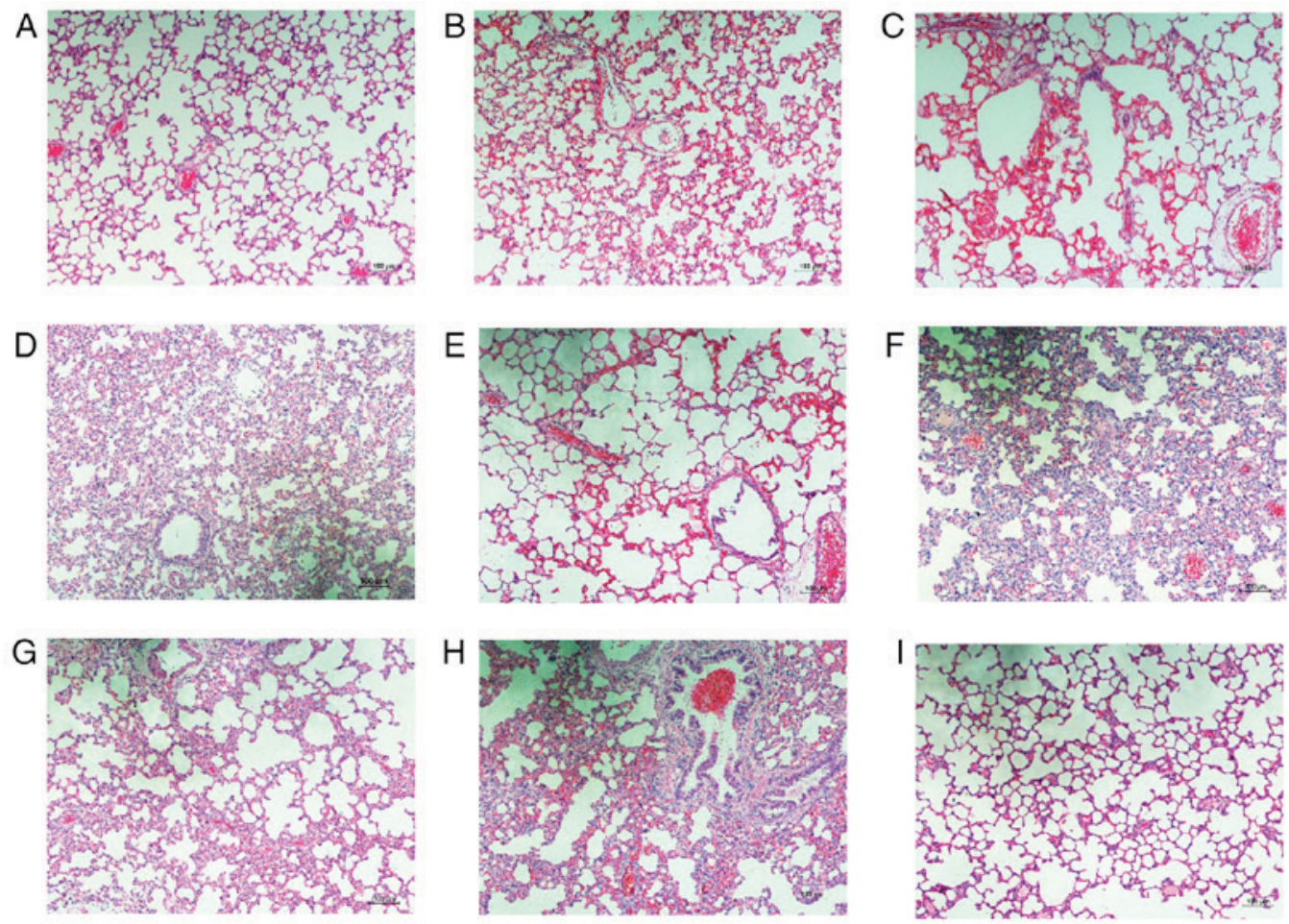

Figure 5. Representative images of H\&E-stained lung tissue from experimental animals. Representative images of lung tissue stained with H\&E are presented for (A) control group at $24 \mathrm{~h}$, (B) LPS-stimulated group at $6 \mathrm{~h},(\mathrm{C})$ BMSC-treated group at $6 \mathrm{~h}$, (D) LPS-stimulated group at $12 \mathrm{~h}$, (E) BMSC-treated group at $12 \mathrm{~h},(\mathrm{~F})$ LPS-stimulated group at $24 \mathrm{~h},(\mathrm{G})$ BMSC-treated group at $24 \mathrm{~h},(\mathrm{H})$ LPS-stimulated group at $48 \mathrm{~h}$ and (I) BMSC-treated group at $48 \mathrm{~h}$. Scale bar, $100 \mu \mathrm{m}$. Magnification, $\mathrm{x} 100, \mathrm{n}=4$ per group. H\&E, hematoxylin and eosin; LPS, lipopolysaccharide; BMSC, bone marrow mesenchymal stem cells.

CD11c and CD206 are specific and widely used markers of M1 and M2 macrophages, respectively, and these two surface molecules were selected in the present study to represent M1 and M2 macrophages (6).

In the present study, the in vitro co-culture experiments demonstrated that macrophages stimulated by LPS expressed higher levels of CD11c and iNOS, in addition to increased secretion of the proinflammatory cytokine TNF- $\alpha$. However, these observations were reversed following direct co-culture of macrophages with BMSCs, and increased levels of Arg-1, IL-10 and TGF- $\beta$ were also reported in the direct co-culture group compared with LPS stimulation of macrophages alone. Liu et al (11) reported that TNF- $\alpha$ release was the standard response of LPS-stimulated macrophages and has a central role in death caused by endotoxemic shock. The results of the current study demonstrated that LPS stimulation of macrophages resulted in a rapid increase in the levels of TNF- $\alpha$, with a marginal repression by BMSCs as early as 3, 7 and $12 \mathrm{~h}$ after LPS stimulation. However, after 24,48 and $72 \mathrm{~h}$, direct co-culture of macrophages with BMSCs led to significant reductions in the TNF- $\alpha$ levels, compared with the stimulation of macrophages alone with LPS. In addition, at the majority of time-points, the levels of IL-10 and TGF- $\beta$ peaked in the group of macrophages that were directly co-cultured with BMSCs. These results illustrated that LPS promoted the ratio of M1-polarized macrophages and increased the secretion of proinflammatory cytokines; however, the presence of BMSCs inhibited such alterations and increased the differentiation of M2 macrophages when co-cultured with macrophages directly. This indicates that the interaction between BMSCs and macrophages may be due to cell-to-cell contact, rather than paracrine cytokines, which differs from previous reports (16,33-35). We hypothesize that this may due to the fact that, after LPS stimulation for $12 \mathrm{~h}$, the original medium was replaced by the normal complete medium, and cytokines in supernatants were subsequently replaced, which may cause the paracrine effect to be less obvious, thus the effect of direct cell-cell contact effect in the experiment played a major role relatively. Western blot analysis and ELISA results demonstrated that macrophages in the BMSC treatment group expressed M2 markers, which was inconsistent with the results of flow cytometry. A number of factors may explain these inconsistencies. Firstly, the polarization of macrophages was a continuous process, with the M1 and M2 phenotypes being two extreme examples. At any stage of the polarization of macrophages towards M1 or M2 phenotypes, there may be a process of secretion of special proteins and cytokines by macrophages during polarization. LPS activated macrophages strongly and an M1 phenotype was induced. However, surface markers for M2 were not detected in the present research. In addition, the present study employed different compared with other studies. The RAW264.7 cell line has been employed in other reports $(18,19)$, which may not reflect the real in vivo situation as they have been previously modified. In the current study, the peritoneal macrophages used differ from RAW264.7 cells as they cannot be induced to polarize towards the M2 phenotype, and the use of peritoneal macrophages in the present study primarily reflected alterations in the physiological functions of macrophages in response to external stimuli. Furthermore, different types of antibodies were employed in the current study; our experimental team searched a number of antibody manufacturers and did not locate a direct labelled antibody for CD206, which was the 

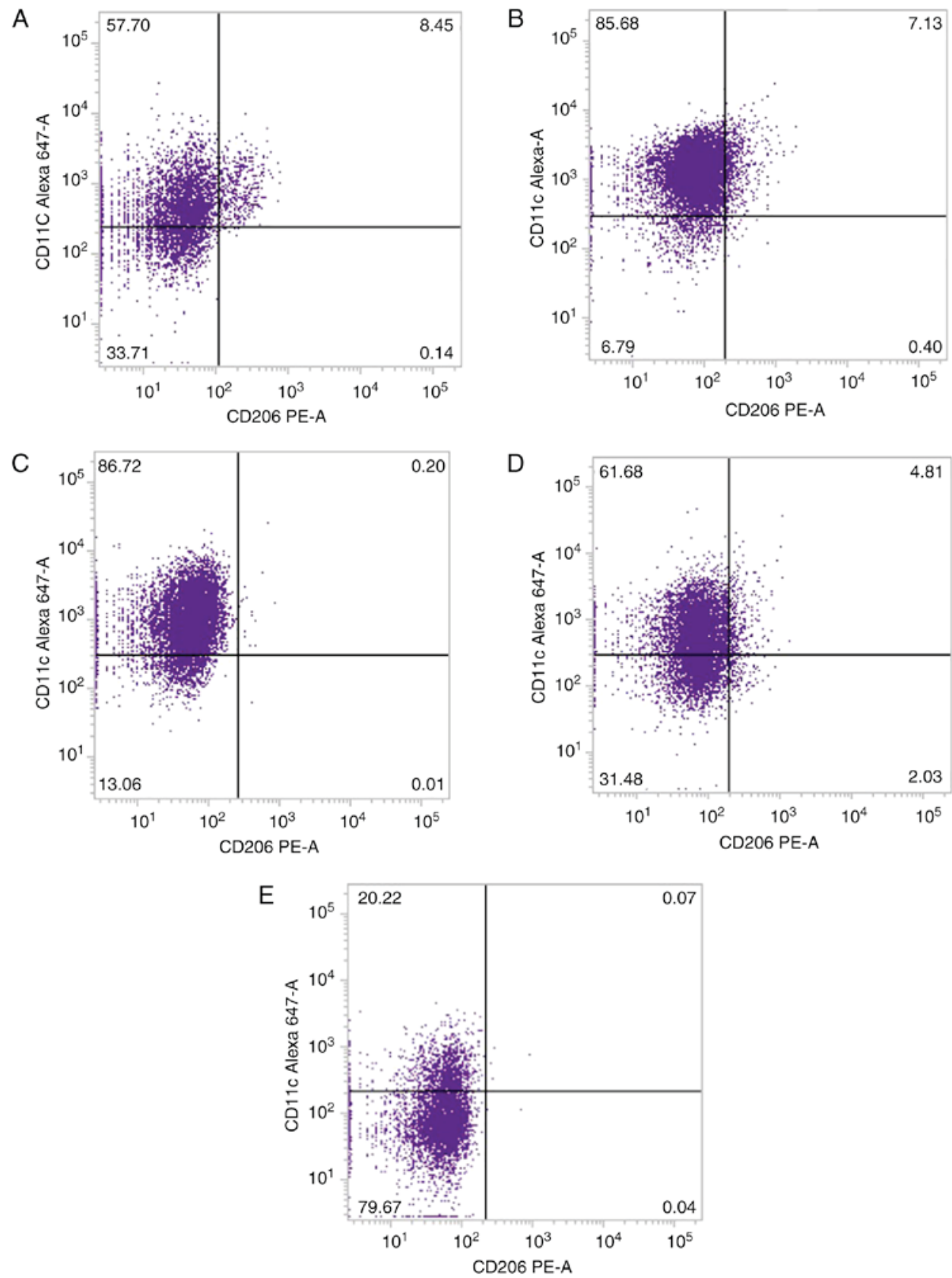

Figure 6. Representative flow cytometry scatter plots for peritoneal macrophages isolated from rats in vivo. (A) Expression of CD11c and CD206 in the normal control group. (B) Expression of CD11c and CD206 on macrophages isolated from rats $24 \mathrm{~h}$ after stimulation with LPS. (C) Expression of CD11c and CD206 on macrophages isolated from BMSC-treated rats $24 \mathrm{~h}$ after LPS treatment. (D) Expression of CD11c and CD206 on macrophages isolated from rats $48 \mathrm{~h}$ after stimulation with LPS. (E) Expression of CD11c and CD206 on macrophages isolated from BMSC-treated rats $48 \mathrm{~h}$ after stimulation with LPS. LPS, lipopolysaccharide; BMSC, bone marrow mesenchymal stem cells; Alexa 647, Alexa Fluor 647; PE, phycoerythrin.

most suitable type for the experiment, and an indirect antibody was employed to mark CD206, which meant the efficiency of labeling was lower, while direct labeling was used to detect the expression of other cell surface markers.

The histopathology determined by H\&E staining of lungs from septic rats demonstrated that, $12 \mathrm{~h}$ after LPS injection, the inflammatory response in lung tissues was increased, which was confirmed by inflammatory cell infiltration, thickened lung interstitium and decreased alveolar space, indicating that lungs suffered from sepsis. However, injection of BMSCs alleviated the pathological changes. This was similar to previous findings that stem cells reduced inflammation in various organs $(18,36,37)$. In the present study, flow cytometry results demonstrated that LPS induced macrophages to differentiate into M1 macrophages after $24 \mathrm{~h}$, and the polarization was not be reversed by BMSCs at the early stage. However, by $48 \mathrm{~h}$, the expression of CD11c returned to a normal level in the LPS-stimulated group, while rats injected with BMSCs exhibited significantly reduced polarization of macrophages into the M1 phenotype, compared with the LPS-stimulated group. However, BMSC injection did not promote the polarization of macrophages into the M2 phenotype when examined at 24 or $48 \mathrm{~h}$. These results indicate that BMSCs may decrease the inflammatory response in the lung tissues and inhibit the polarization of peritoneal macrophages into an M1 phenotype. The above results were consistent with previous that investigate 
whether the injection of BMSCs in animals may reduce the inflammatory response in various diseases $(18,24,27,37,38)$.

Investigating the effect of BMSCs on alterations in macrophage polarization in vivo and in vitro may provide the basis for the future application of BMSCs in the treatment of different diseases. Due to the complexity of the immune system, the mechanism of BMSCs in in vivo treatment are associated with more complex regulatory networks and specific factors than in vitro, and its mechanism for initiation and termination remain unclear. However, stem cells are associated with their own limitations and safety issues. The strict requirements for donor age and the insufficient systematic delivery of MSCs to target tissues limits the use of stem cells (39). In the present study, animal model results indicated that BMSCs may have great potential, however, rat studies may not be identical with humans. Further studies overcoming the application of MSCs are required.

In conclusion, the results of the present study demonstrated that BMSCs co-cultured directly with macrophages prevented LPS-stimulated macrophages from differentiating into an M1 phenotype, as determined by the reduced expression of CD11c in peritoneal macrophages and the reduced levels of inflammatory cytokines released. Furthermore, in vivo results indicated that BMSCs exhibited therapeutic effects on experimental sepsis by reducing the pathological inflammatory response in the lung tissues.

\section{Acknowledgements}

The present study was supported by the National Natural Science Foundation of China (grant nos. 81271329 and 81471237) and the Medical Scientific Research Foundation of Guangdong Province of China (grant no. A2016108). The authors gratefully acknowledge Dr Stanley Lin (Shantou University, Shantou, China) for his assistance with the English drafting of the manuscript.

\section{References}

1. Annane D, Bellissant E and Cavaillon JM: Septic shock. Lancet 365: 63-78, 2005.

2. Singer M, Deutschman CS, Seymour CW, Shankar-Hari M, Annane D, Bauer M, Bellomo R, Bernard GR, Chiche JD, Coopersmith CM, et al: The third international consensus definitions for sepsis and septic shock (Sepsis-3). Jama 315: 801-810, 2016.

3. Cohen J, Vincent JL, Adhikari NK, Machado FR, Angus DC, Calandra T, Jaton K, Giulieri S, Delaloye J, Opal S, et al: Sepsis: A roadmap for future research. Lancet Infect Dis 15: 581-614, 2015.

4. Seymour CW, Rea TD, Kahn JM, Walkey AJ, Yealy DM and Angus DC: Severe sepsis in pre-hospital emergency care: Analysis of incidence, care and outcome. Am J Respir Crit Care Med 186: 1264-1271, 2012.

5. Wynn TA, Chawla A and Pollard JW: Macrophage biology in development, homeostasis and disease. Nature 496: 445-455, 2013.

6. Zhang X, Goncalves R and Mosser DM: The isolation and characterization of murine macrophages. Curr Protoc Immunol: Chapter 14, Unit 14.1, 2008.

7. Ying W, Cheruku PS, Bazer FW, Safe SH and Zhou B: Investigation of macrophage polarization using bone marrow derived macrophages. J Vis Exp, 2013.

8. Ivashkiv LB: Epigenetic regulation of macrophage polarization and function. Trends Immunol 34: 216-223, 2013.

9. Ka MB, Daumas A, Textoris J and Mege JL: Phenotypic diversity and emerging new tools to study macrophage activation in bacterial infectious diseases. Front Immunol 5: 500, 2014.
10. López-Bojórquez LN, Dehesa AZ and Reyes-Terán G: Molecular mechanisms involved in the pathogenesis of septic shock. Arch Med Res 35: 465-479, 2004.

11. Liu LL, Gong LK, Wang H, Xiao Y, Wu XF, Zhang YH, Xue X, Qi XM and Ren J: Baicalin inhibits macrophage activation by lipopolysaccharide and protects mice from endotoxin shock. Biochem Pharmacol 75: 914-922, 2008.

12. Simovart HE, Põldoja E, Kokk K, Tapfer H, Liigant A, Talvik R and Roosaar P: Changes of activated macrophages and apoptotic cell count in the organs of rats during experimental sepsis. Medicina (Kaunas) 39: 932-939, 2003.

13. Prockop DJ and Oh JY: Mesenchymal stem/stromal cells (MSCs): Role as guardians of inflammation. Mol Ther 20: 14-20, 2012.

14. Soleimani M and Nadri S: A protocol for isolation and culture of mesenchymal stem cells from mouse bone marrow. Nat Protoc 4: 102-106, 2009

15. Jin HJ, Bae YK, Kim M, Kwon SJ, Jeon HB, Choi SJ, Kim SW, Yang YS, Oh W and Chang JW: Comparative analysis of human mesenchymal stem cells from bone marrow, adipose tissue, and umbilical cord blood as sources of cell therapy. Int J Mol Sci 14: 17986-18001, 2013.

16. Zhang QZ, Su WR, Shi SH, Wilder-Smith P, Xiang AP, Wong A, Nguyen AL, Kwon CW and Le AD: Human gingiva-derived mesenchymal stem cells elicit polarization of $\mathrm{m} 2$ macrophages and enhance cutaneous wound healing. Stem Cells 28: 1856-1868, 2010.

17. Wise AF, Williams TM, Kiewiet MB, Payne NL, Siatskas C, Samuel CS and Ricardo SD: Human mesenchymal stem cells alter macrophage phenotype and promote regeneration via homing to the kidney following ischemia-reperfusion injury. Am J Physiol Renal Physiol 306: F1222-F1235, 2014.

18. Geng Y, Zhang L, Fu B, Zhang J, Hong Q, Hu J, Li D, Luo C, Cui S, Zhu F and Chen X: Mesenchymal stem cells ameliorate rhabdomyolysis-induced acute kidney injury via the activation of M2 macrophages. Stem Cell Res Ther 5: 80, 2014.

19. Gao S, Mao F, Zhang B, Zhang L, Zhang X, Wang M, Yan Y, Yang T, Zhang J, Zhu W, et al: Mouse bone marrow-derived mesenchymal stem cells induce macrophage M2 polarization through the nuclear factor- $\mathrm{\kappa B}$ and signal transducer and activator of transcription 3 pathways. Exp Biol Med (Maywood) 239: 366-375, 2014.

20. Ray A and Dittel BN: Isolation of mouse peritoneal cavity cells. J Vis Exp 28: pii: 1488, 2010

21. Chai X, Guo Y, Jiang M, Hu B, Li Z, Fan J, Deng M, Billiar TR, Kucera HR, Gaikwad NW, et al: Oestrogen sulfotransferase ablation sensitizes mice to sepsis. Nat Commun 6: 7979, 2015.

22. Zheng G, Ge M, Qiu G, Shu Q and Xu J: Mesenchymal stromal cells affect disease outcomes via macrophage polarization. Stem Cells Int 2015: 989473, 2015.

23. Kim J and Hematti P: Mesenchymal stem cell-educated macrophages: A novel type of alternatively activated macrophages. Exp Hematol 37: 1445-1453, 2009.

24. Lee JW, Gupta N, Serikov V and Matthay MA: Potential application of mesenchymal stem cells in acute lung injury. Expert Opin Biol Ther 9: 1259-1270, 2009.

25. Murray PJ and Wynn TA: Obstacles and opportunities for understanding macrophage polarization. J Leukoc Biol 89: 557-563, 2011.

26. Abumaree MH, Al Jumah MA, Kalionis B, Jawdat D, Al Khaldi A, Abomaray FM, Fatani AS, Chamley LW and Knawy BA: Human placental mesenchymal stem cells (pMSCs) play a role as immune suppressive cells by shifting macrophage differentiation from inflammatory M1 to anti-inflammatory M2 macrophages. Stem Cell Rev 9: 620-641, 2013.

27. Mei SH, Haitsma JJ, Dos Santos CC, Deng Y, Lai PF, Slutsky AS, Liles WC and Stewart DJ: Mesenchymal stem cells reduce inflammation while enhancing bacterial clearance and improving survival in sepsis. Am J Respir Crit Care Med 182: 1047-1057, 2010.

28. Zenker S, Panteleev-Ivlev J, Wirtz S, Kishimoto T, Waldner MJ, Ksionda O, Tybulewicz VLJ, Neurath MF and Atreya I: A key regulatory role for Vavl in controlling lipopolysaccharide endotoxemia via macrophage-derived IL-6. J Immunol 192: 2830-2836, 2014.

29. Boomer JS, To K, Chang KC, Takasu O, Osborne DF, Walton AH, Bricker TL, Jarman SD II, Kreisel D, Krupnick AS, et al: Immunosuppression in patients who die of sepsis and multiple organ failure. Jama 306: 2594-2605, 2011.

30. Fang SH, Hou YC, Chang WC, Hsiu SL, Lee Chao PD and Chiang BL: Morin sulfates/glucuronides exert anti-inflammatory activity on activated macrophages and decreased the incidence of septic shock. Life Sci 74: 743-756, 2003. 
31. Ayala A, Elphick GF, Kim YS, Huang X, Carreira-Rosario A, Santos SC, Shubin NJ, Chen Y, Reichner J and Chung CS Sepsis-induced potentiation of peritoneal macrophage migration is mitigated by programmed cell death receptor-1 gene deficiency. J Innate Immun 6: 325-338, 2014.

32. Oh JY, Ko JH, Lee HJ, Yu JM, Choi H, Kim MK, Wee WR and Prockop DJ: Mesenchymal stem/stromal cells inhibit the NLRP3 inflammasome by decreasing mitochondrial reactive oxygen species. Stem Cells 32: 1553-1563, 2014.

33. Gotts JE and Matthay MA: Mesenchymal stem cells and acute lung injury. Crit Care Clin 27: 719-733, 2011.

34. Zhao G, Miao H, Li X, Chen S, Hu Y, Wang Z and Hou Y: TGF-beta3-induced miR-494 inhibits macrophage polarization via suppressing PGE2 secretion in mesenchymal stem cells FEBS Lett 590: 1602-1613, 2016.

35. Luz-Crawford P, Djouad F, Toupet K, Bony C, Franquesa M, Hoogduijn MJ, Jorgensen C and Noël D: Mesenchymal stem cell-derived interleukin 1 receptor antagonist promotes macrophage polarization and inhibits B cell differentiation. Stem Cells 34: 483-492, 2016.

36. Nemeth K, Leelahavanichkul A, Yuen PS, Mayer B, Parmelee A, Doi K, Robey PG, Leelahavanichkul K, Koller BH, Brown JM, et al: Bone marrow stromal cells attenuate sepsis via prostaglandin E (2)-dependent reprogramming of host macrophages to increase their interleukin-10 production. Nat Med 15: 42-49, 2009.
37. Zhu YG, Feng XM, Abbott J, Fang XH, Hao Q, Monsel A, Qu JM, Matthay MA and Lee JW: Human mesenchymal stem cell microvesicles for treatment of Escherichia coli endotoxin-induced acute lung injury in mice. Stem Cells 32: 116-125, 2014.

38. Weil BR, Herrmann JL, Abarbanell AM, Manukyan MC, Poynter JA and Meldrum DR: Intravenous infusion of mesenchymal stem cells is associated with improved myocardial function during endotoxemia. Shock 36: 235-241, 2011.

39. Bernardo ME and Fibbe WE: Mesenchymal stromal cells: Sensors and switchers of inflammation. Cell Stem Cell 13: 392-402, 2013

(7) (3) $(9)$ This work is licensed under a Creative Commons International (CC BY-NC-ND 4.0) License. 